\title{
Maxillary Sinusitis
}

\author{
Kandasamy Ganesan and Neelam Rathod
}

\subsection{Introduction}

Sinusitis, also known as rhinosinusitis with the association of rhinitis, is a common presentation within the primary care setting, due to the effect it can have on individuals' quality of life. It is defined as the inflammation of the mucosal lining in at least one of the paranasal sinuses with an acute presentation lasting for less than 12 weeks and chronic extending to durations greater than this. The maxillary sinus is the largest of four paranasal sinuses and, with its close relationship to the underlying dentition, it is often susceptible to inflammatory processes. This chapter explores the anatomy, physiology of the maxillary sinus, the aetiology, assessment and management of maxillary sinusitis.

\subsection{Anatomy of Maxillary Sinus}

The maxillary sinus is the largest of the paranasal sinuses and develops during day 65-75 of gestation, with a volume of approximately $1 \mathrm{~cm}^{3}$ at birth. Up untill an average age of 12 , pneumatisation of the maxillary sinus continues as the space occupied by tooth germs is freed through their eruption [1]. In the latter stages of development, it pneumatises inferiorly, guided by the eruption pathway of the permanent dentition.

The main function of the maxillary sinus is involved in the humidification and warming of inspired air as well as prevention of microorganism ingress through mucociliary action. In addition to this, the paranasal sinus contributes to voice reso-

K. Ganesan $(\varangle)$

Department of Oral and Maxillofacial Surgery, Southend

University Hospitals NHS Trust, Southend-on-Sea, UK

University of Leeds, Leeds, UK

e-mail: mailme@kandyganesan.com

N. Rathod

Department of OMFS, Southend University Hospital NHS Trust,

Southend-on-Sea, UK

e-mail: n.rathod@nhs.net nance. A possible evolutionary function may also be as a "crumple zone' during trauma, thus protecting the brain (Fig. 23.1).

In an adult, the maxillary sinus takes the form of a quadrangle pyramidal shape, with the base adjacent to the nasal cavity and the peak extending towards the zygomatic process, and a volume in the region of $15 \mathrm{~cm}^{3}$. The roof of the sinus is formed of the orbital floor in the centre of which runs the infraorbital neurovascular bundle. The anterior wall of the maxillary sinus is the weakest of the walls, with the thinnest section superior to the canine resulting in the canine fossa. It is also perforated by the infraorbital nerve that supplies the maxillary sinus, along with the greater palatine nerve. The posterior wall of the sinus lies in front of and shelters the internal maxillary artery, sphenopalatine artery, Vidian canal and the greater palatine nerve. The inferior wall is the most varying in shape, with invaginations corresponding to the alveolar bone of the maxilla; the anatomical root structures of maxillary molars and the hard palate. The bone separating the dental roots can be varying

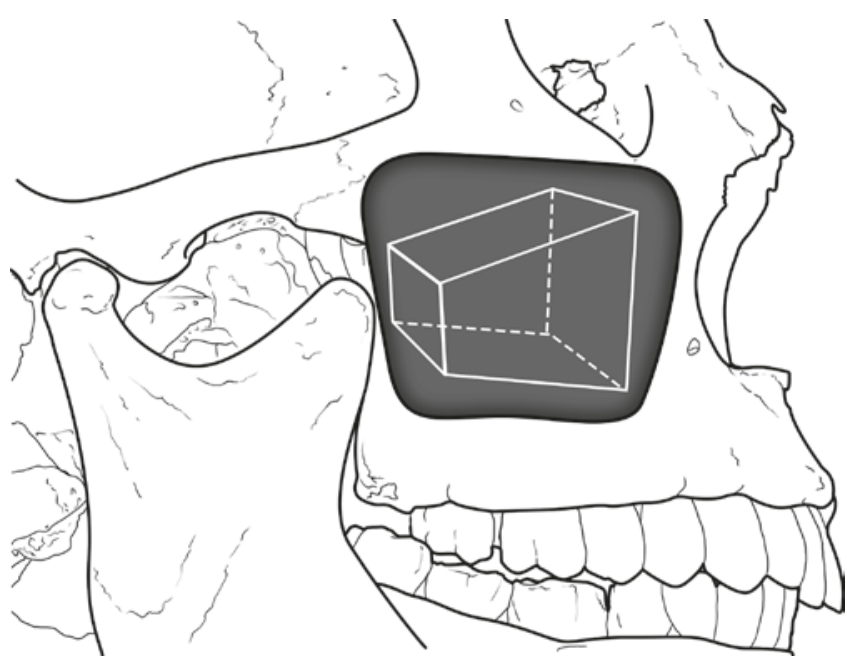

CAssociation of Oral and Maxillofacial Surgeons of India

Fig. 23.1 Schematic diagram of the maxillary sinus with representation of the geometric shape of the cavity 
in its quantity; from complete absence to thicknesses of up to $12 \mathrm{~mm}$ [2]. This close proximity is a contributing factor towards the likelihood of dental iatrogenic and inflammatory pathology presenting in the maxillary sinus. The medial wall that is parallel to the nasal cavity communicates with it through the natural sinus ostium at the postero-superior aspect of sinus. This ostium opens in to a triangular space of approximately $15 \mathrm{~mm}^{2}$ diameter formed by the uncinate process medially, the lamina papyracea laterally and the ethmoidal bulla posteriorly before

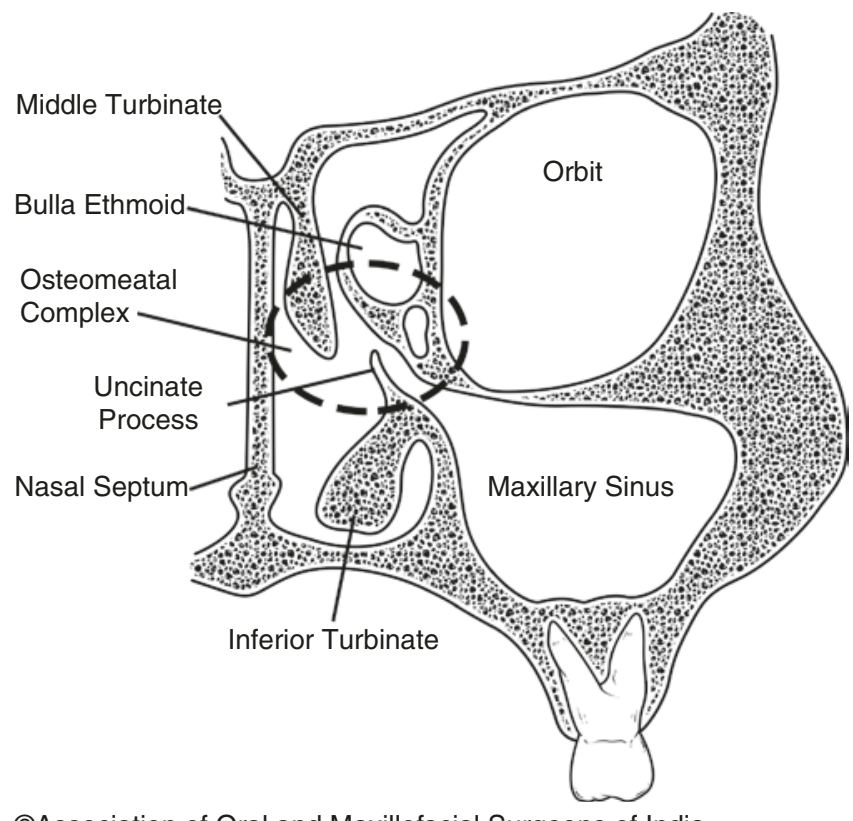

CAssociation of Oral and Maxillofacial Surgeons of India

Fig. 23.2 Schematic diagram showing a coronal cross section of the maxillary sinus and the osteomeatal complex communicating with the nasal cavity through a semilunar hiatus in the middle meatus. This is an area of common drainage from the maxillary, anterior ethmoidal and frontal sinuses. Some individuals may exhibit anterior/posterior fontanelles; bony dehiscences inferior to this, covered with mucosa. These can act as accessory ostia, points of drainage when the main osteomeatal complex is blocked or there is a change in sinus pressure. An osteomeatal complex (OMC) is an important functional unit and is also a key area for the pathogenesis of chronic rhinosinusitis. It consists of multiple bony structures, air spaces and ostia. The bony structures include the middle turbinate, uncinate process of the ethmoid and bulla of the ethmoid. Air spaces are formed by the frontal recess, infundibulum of the ethmoid and the middle meatus. Ostia consists of anterior ethmoid, maxillary and frontal sinuses. The classic OMC as mentioned above has been described as the anterior osteomeatal unit. The sphenoethmoidal recess and the superior meatus are referred to as the posterior meatal unit (Fig. 23.2).

Maxillry sinus septa, present in approximately $28.4 \%$ of cases [3], are thin projections of cortical bone that divide the sinus into more than one compartment either in the transverse, sagittal or horizontal plane, usually in the region of the first or second molar.

Smaller air-filled compartments in the periphery of the maxillary sinus can include Haler cells and Concha bullosa. Haller cells are air cavities encompassed in the ethmoidal capsule and are located below the inferomedial aspect of the orbital floor, and lateral to the ethmoidal cells. These can be present in a range of sizes as well as unilaterally or bilaterally. Concha bullosa is another variant air-filled cavity pneumatised in to the middle turbinate. These particular anatomical (Fig. 23.3) varia-
Fig. 23.3 Schematic diagram with a sagittal cross section of the nasal cavity and the respective openings in to it

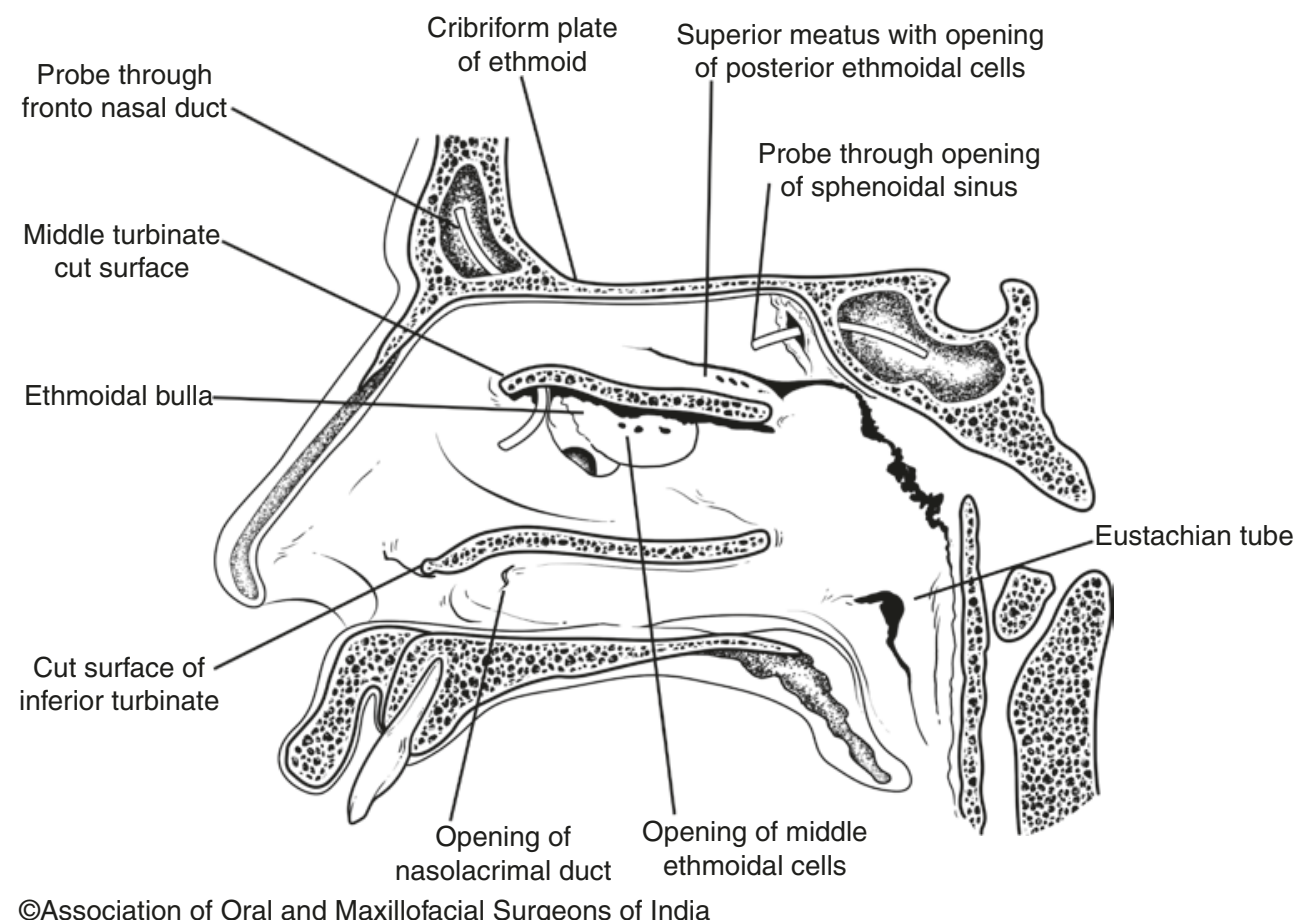


Fig. 23.4 Schematic diagram of the secretary and clearance of the mucous via the mucociliary cells

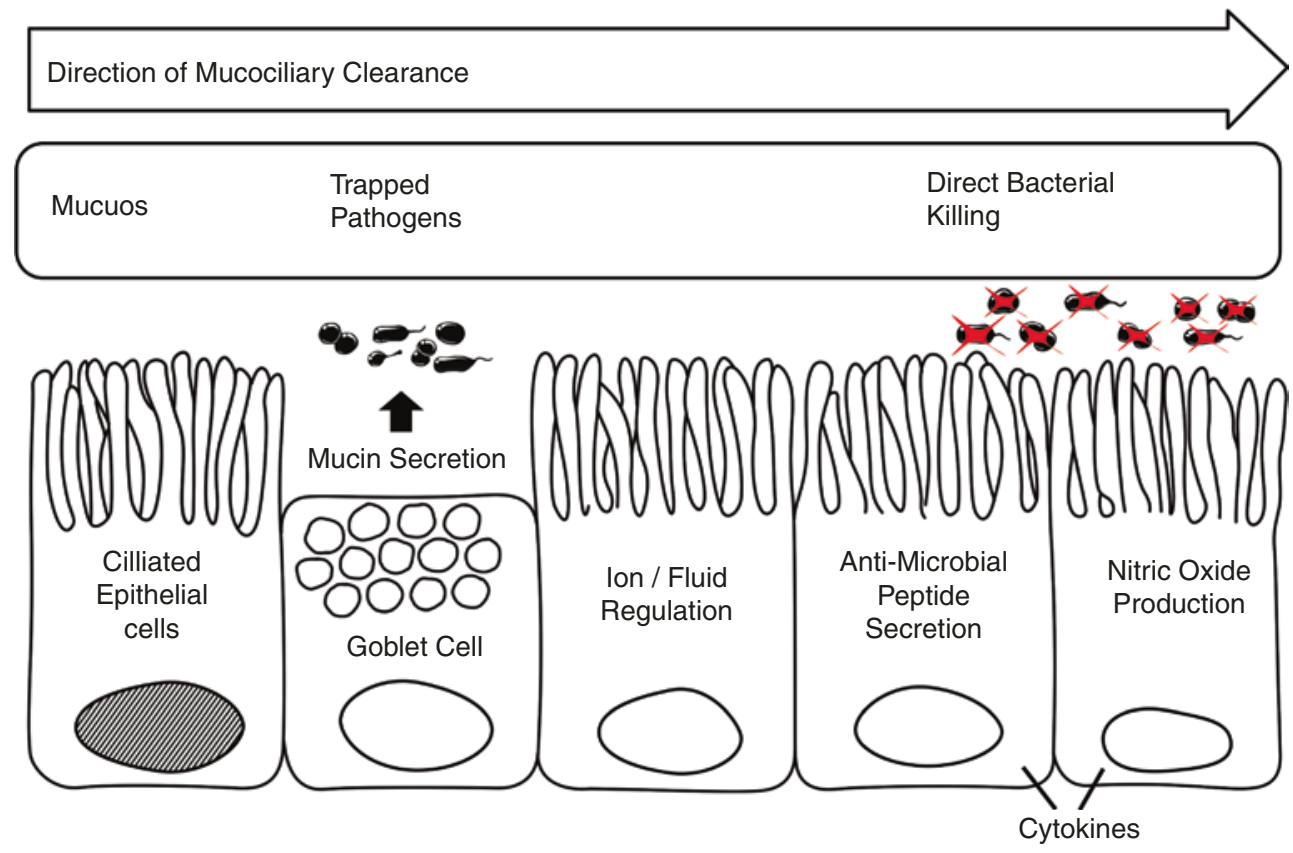

(C)Association of Oral and Maxillofacial Surgeons of India tions have the potential to influence the dimensions of the osteomeatal complex, increasing the likelihood of sinus disease [4] .

\subsection{Maxillary Sinus Physiology}

At a histological level, the maxillary sinus consists of ciliated columnar cells, basal cells and goblet cells and thus forming respiratory epithelium architecture. Unlike the rest of the respiratory pathway, the sinuses have fewer ciliated and goblet cells; friable epithelium and fewer seromucous cells, making them prone to microorganism ingress and related pathology.

The clearance of secretions from the sinuses is through a combination of ciliary and mucous action. The mucous secreted by the goblet cells consists of $96 \%$ water, with the rest consisting of glycoproteins, immunoglobulins, histamines, lactoferrin, prostaglandins and lysozymes [5]. It functions to trap foreign body and defend against bacterial ingress. This is combined with the ciliary action that promotes a spiral action of movement of the mucous through active transport from the base of the sinus towards the natural ostium in the supero-posterior aspect of the medial sinus wall and thus working against the forces of gravity.

Normal flora within the maxillary sinus is usually a combination of sterile aerobic and anaerobic organisms including bacteria organised in a complex biofilm within the sinus mucus layer. These usually include mainly aerobic B-hemolytic streptococci, staphylococci and haemophilus spp. The anaerobic organisms, that are fewer in quantity, include peptostreptococcus, fusobacterium $s p$ and bacteroides. Whether these organisms are present in normal physiol- ogy or transiently is poorly understood. Host specific and non-specific defence against these bacterial species is also part of normal physiology with the sinus mucosa producing secretion including antimicrobial peptides, proteins, neutrophils, macrophages coupled with the mucociliary transport towards the ostium (Fig. 23.4).

\subsection{Sinusitis Pathophysiology}

Due to the narrow size of the ostium opening, occlusion and related pathology of the maxillary sinus space are very likely possibilities. Obstruction of the sinus ostium can either be primary to the sinusitis process or secondary inflammation originating from elsewhere in the sinus.

With obstruction of the natural ostium, there is reduced oxygenation and gas exchange within the maxillary sinus, alongside reduced mucociliary action and mucous stasis. In the most common acute form of sinusitis, viral upper respiratory tract infections result in significant nasal congestion that results in maxillary sinus blockage and stasis. Anatomical differences such as large middle turbinates, deviated nasal septums or concha bullosa can increase the likelihood of ostium occlusion. Polyps, in particular those originating from the ethmoid, may also obstruct the maxillary sinus opening (Table 23.1).

Any form of obstruction and subsequent stasis in the maxillary sinus produce a favourable environment for the flourishing of an anaerobic environment, encouraging the formation of purulent secretions. In addition to this, changes in the sinus pressure, coupled with local mucosal inflammation, contribute to the symptoms of pain and pressure in the region [6]. The initial lower sinus pressure is caused by the 
Table 23.1 Membrane swelling leading to sinus infection

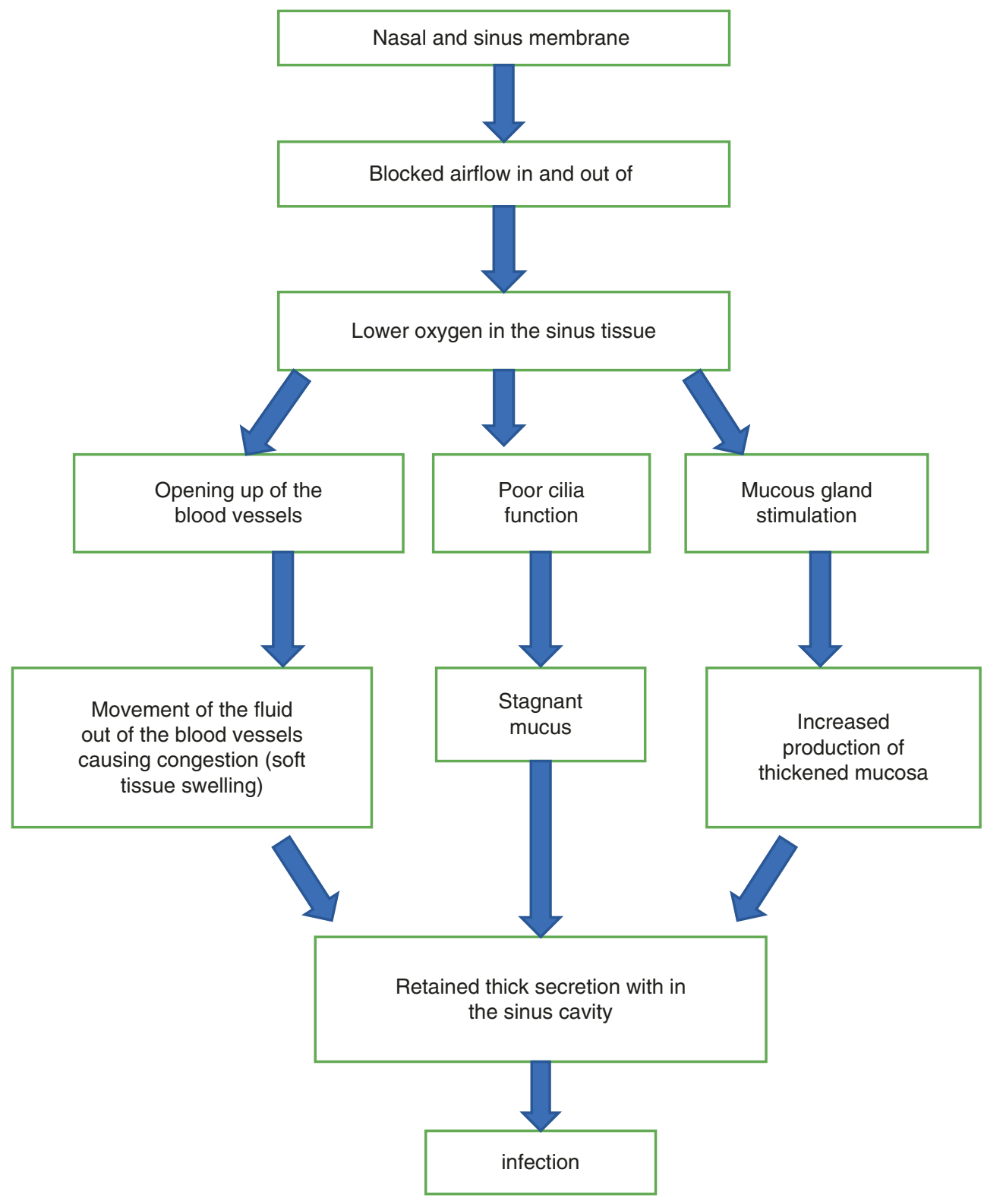

consumption of the finite oxygen within the blocked and confined maxillary sinus cavity. This is followed by a transient increase in pressure where there is a greater production of carbon dioxide and sinus secretion.

Chronic rhinosinusitis has features of long-term inflammation, rather than primary infection, of the nasal passages and paranasal sinuses with an unknown underlying cause. It is likely to be part of a cycle involving inflammation, infection and subsequent obstruction of the ostium [7]. Without the presence of polyps, chronic sinusitis may be multi-factorial in nature, with one or several predisposing factors as outlined below.

Nasal polyps are oedematous masses of the mucosal membrane found in the nasal passages and paranasal sinuses. Histologically, they show squamous epithelial proliferation, a thickened basement membrane, absence of neurosensory filaments and inflammatory cell infiltrate with high numbers of oesinophils [8]. The pathogenesis in the formation of polyps and its contribution towards chronic rhinosinusitis is poorly understood. One in vivo study involving rabbits with stimulated maxillary sinusitis observed the formation of inflammatory-type polyps in subjects with purulent infection and granulation based polyps in both purulent and nonpurulent infections. In both infective processes, epithelial damage to the mucosal lining appeared to be a significant factor in the initiation of polyp formation [9]. Other evidence shows that high levels of interleukin 5, 13 and histamines in the polypoid tissue may also have a contributing role [10]. The general consensus of studies shows a high level of inflammatory mediators in the initiation and presence of nasal polyps, suggesting chronic inflammation to be a key factor. 


\subsubsection{Microorganisms of Sinusitis}

The polymicrobial nature of maxillary sinusitis has been well documented. The initial aerobic bacterial infective organisms often include Streptococcus pneumoniae, Haemophilus influenzae and Moraxella catarrhalis in acute sinusitis [11]. Chronic sinusitis has found to harbour the aforementioned organisms in addition to Prevotella species, Fusobacterium species and anaerobic streptococci [12]. More recent studies have cultured Staphylococcus aureus, in particular methicillin resistant-type from sinus mucosal samples [13].

\subsection{Predisposing Factors}

\subsubsection{Anatomical Variation}

Any variation deemed to alter the volume or the size of the maxillary ostium may contribute to an increase risk of sinusitis. This includes [14]:

- Haller cells.

- Concha bullosa.

- Reduced infundibular width.

- Septal deviation.

- Choanal atresia.

- Nasal polyps.

- Hypoplasia of the sinus.

- Maxillary dentition roots in the sinus.

\subsubsection{Atopy (Allergy)}

The distinct relationship between the allergy and inflammation remains unclear. Current hypothesis predicts that with the ventilation passage in continuum with the nasal mucosa, inflammation along the rest of the airway can affect the nasal passages, thus causing narrowing of the ostium. This is supported by the high incidence of chronic rhinosinusitis with allergies driven by $\operatorname{IgE}$ mediators.

\subsubsection{Asthma}

The association between chronic sinusitis, mainly with nasal polyps, and asthma has been well documented. Several studies have shown radiographic sinus mucosal abnormalities in individuals with asthma, in particular with those suffering from severe steroid-dependent asthma [15]. Similar studies show a trend in the severity of sinusitis correlating to the severity of asthma experienced, supporting the theory of inflammation of a unified mucosal airway concept of the dis- ease process. The association between asthma and sinusitis is further indicated with studies showing individuals reporting improvement in their asthma symptoms subsequent to medical and/or surgical treatment of their sinusitis [16].

\subsubsection{Aspirin}

Aspirin/NSAID hypersensitivity has been found to have an association with a persistent form of chronic sinusitis, usually with the presence of nasal polyps. Along with severe asthma, this disease process has been named the 'Aspirintriad'. The mechanism of pathogenicity is thought to be linked to cyclooxygenase inhibition and inhibition of arachnoid acid metabolism rather than an underlying immunological process. The presence of hyperplastic sinus mucosa contributes to a high level of recurrence in nasal polyps subsequent to sinus surgery for removal [14].

\subsubsection{Environmental}

Several environmental factors have been associated with an increased incidence of sinusitis with changes in air quality acting as suggestive stimulants. Greater prevalence has been identified in patients exposed to chemical air pollutants including pharmaceutical products, photocopying ink byproducts, smoke and dampness $[14,17]$.

\subsubsection{Ciliary Impairment}

This may be present in the form of reduced cilia or ciliary cells, causing impairment of mucociliary flow and thus creating environments favourable for bacterial or viral sinusitis.

\subsubsection{Smoking}

Smoking is likely to diminish the presence of normal microbiological flora in the nasal and paranasal spaces, allowing for the growth of pathogenic microorganisms and thus eliciting a hypersensitivity reaction in the sinonasal mucosa [14].

\subsubsection{Gastro-Oesophageal Reflux}

It has been suggested that inflammation may be elicited when the oro- and nasopharynx comes in contact with gastric acids during reflux and subsequently contributing to sinusitis [18]. 


\subsubsection{Odontogenic Maxillary Sinusitis}

As part of the examination process, this should be evaluated at the early stages and could prevent prolonged periods of investigations and symptomatic management (Fig. 23.5). Approximately 15-24\% of unilateral maxillary sinusitis cases are believed to be of odontogenic origin [19], with some studies indicating this figure to be as high as $40 \%$ [20]. In a metaanalysis of 15 observational review studies consisting 770 cases, the first molars were found to be the most common tooth to be causative for odontogenic sinusitis $(22.51 \%)$, followed by third molars $(17.21 \%)$, second molars $(3.97 \%)$, premolars $(5.96 \%)$ and lastly canines $(0.66 \%)$. The etiological contribution to the sinusitis was found most commonly to be from iatrogenic factors $(55.97 \%)$, periodontal disease $(40.38 \%)$ and odontogenic cysts $(6.66 \%)$ [21]. Figure 23.6 outlines the distribution of the iatrogenic processes leading to sinusitis [21].

The disruption in maxillary sinus floor, from whichever odontogenic source, causes localised inflammation that remains persistent in that area or spreads along the sinomucosal surface with subsequent obstruction of the sinus ostium. Bacterial colonisation have been shown to consist of both aerobic and anaerobic species in $75 \%$ of cases, with the other $25 \%$ predominantly anaerobic in nature. Staphylococcus aureus and streptococcus pneumonia were the predominant aerobic bacteria isolated. When looking at the anaerobic bacteria distribution, Peptococcus and Prevotella species dominated and haemophilus and Moraxella species were absent in cases of odontogenic maxillary sinusitis [20].

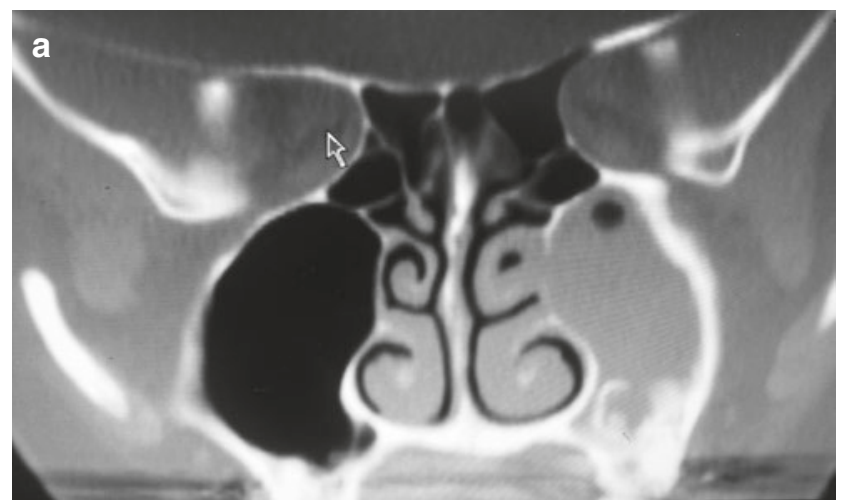

CAssociation of Oral and Maxillofacial Surgeons of India

Fig. 23.5 (a) A coronal section of a CT of maxillary sinus showing extensive left-sided maxillary sinusitis. A periapical radiolucency on the upper left first molar with a breach in the cystic area to allow the pus

\subsection{Classification of Sinusitis}

Sinusitis is defined as an inflammation of the paranasal sinus mucosal lining; however, it rarely presents in isolation and is usually coupled with the inflammation of the nasal mucosa, thus being termed as rhinosinusitis. The cardinal features, of which two are required for a suggestive diagnosis of a rhinosinusitis, are outlined in the 2012 European position paper by the International Rhinology Society (Table 23.2). The condition is broadly classified based upon the chronicity of the condition as well as whether there is presence of polyps.

\subsubsection{Acute Rhinosinusitis}

Acute rhinosinusitis (ARS) is defined as persistent sinusitis that resolves within a 12 -week period. It is a common presentation within the populations globally, with prevalence rates between 6 and $15 \%$ [14].

\subsubsection{Chronic Rhinosinusitis}

Chronic rhinosinusitis is defined by the persistence of sinusitis symptoms for greater than 12 weeks, with no resolution after initial sinusitis treatment. It is further subdivided into whether there is a clinical and radiographic presentation of nasal polyps.

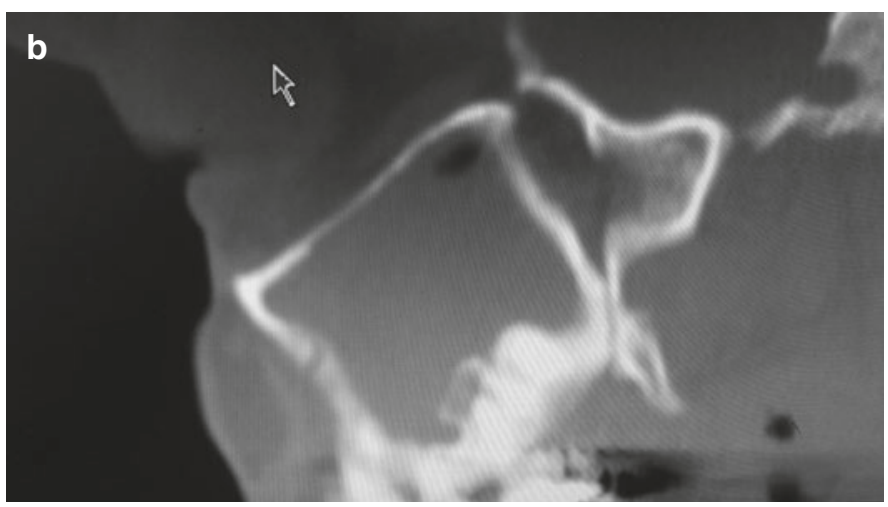

to enter the sinus and causing the sinusitis. (b) A sagittal section same image showing the extent of the sinusitis. A periapical radiolucency is noticeable in relation to mesiobuccal root of upper left first molar 
Fig. 23.6 A pie chart illustrating a distribution of the iatrogenic processes contributing to odontogenic sinusitis [21]

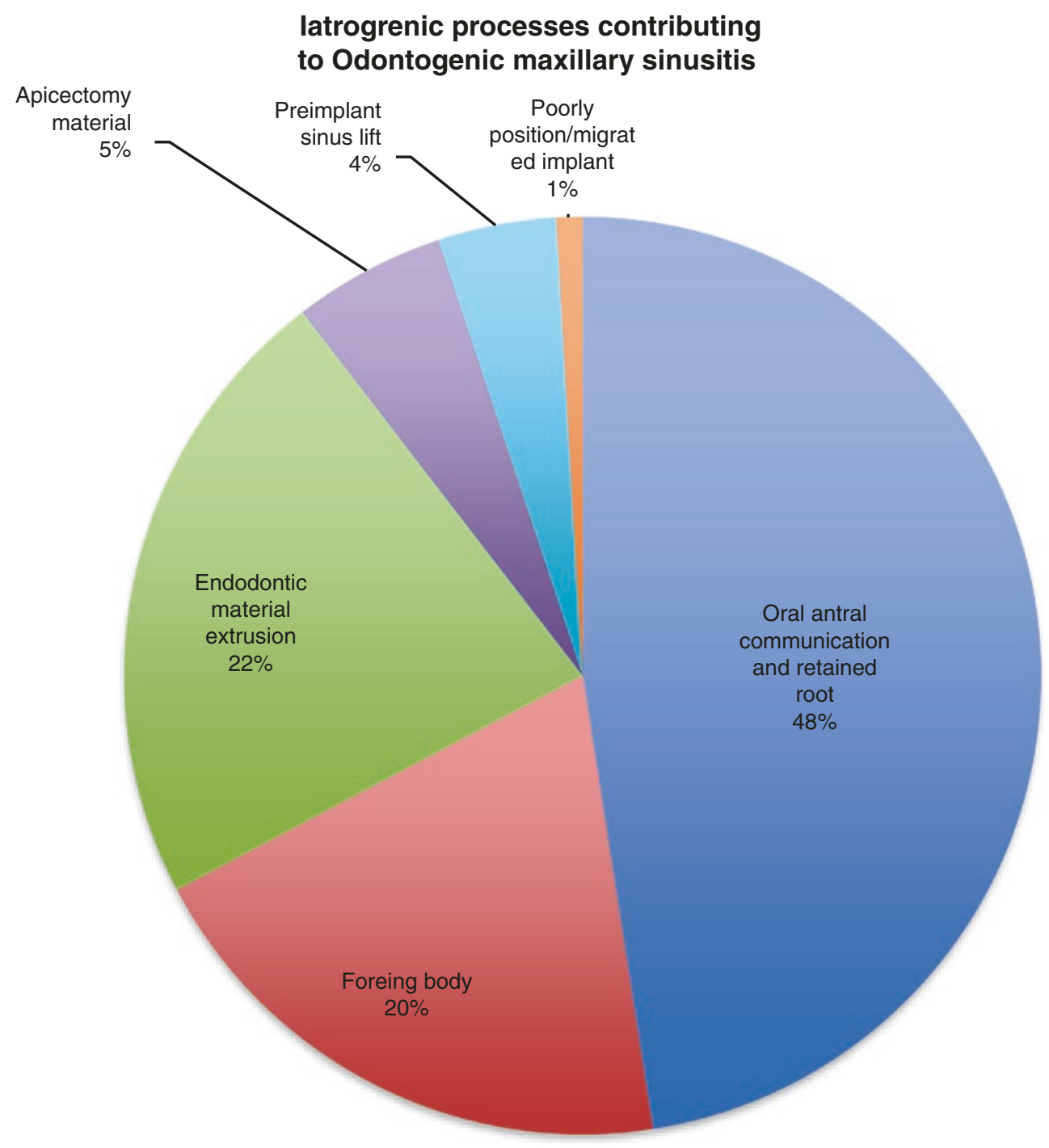

CAssociation of Oral and Maxillofacial Surgeons of India
Table 23.2 Features of rhinosinusitis [14]

\begin{tabular}{|l|l|l|}
\hline Symptoms & Endoscopic signs of & CT changes \\
\hline $\begin{array}{l}\text { Nasal } \\
\text { obstruction/ } \\
\text { blockage }\end{array}$ & $\begin{array}{l}\text { - Nasal polyps, } \\
\text { Mucosal oedema/ } \\
\text { obstruction of the } \\
\text { middle meatus, }\end{array}$ & $\begin{array}{l}\text { Mucosal change } \\
\text { in the sinus }\end{array}$ \\
$\begin{array}{l}\text { Nucosal change } \\
\text { congestion }\end{array}$ & $\begin{array}{l}\text { - Mucopurulent } \\
\text { in the osteomeatal } \\
\text { complex }\end{array}$ \\
$\begin{array}{l}\text { Anterior/ } \\
\text { posterior } \\
\text { nasal } \\
\text { discharge mainly from }\end{array}$ & \\
$\begin{array}{l}\text { Anosmia/ } \\
\text { the middle meatus }\end{array}$ & \\
$\begin{array}{l}\text { Hyposmia } \\
\text { Facial pain/ } \\
\text { pressure }\end{array}$ & & \\
\hline
\end{tabular}

\subsubsection{Fungal Rhinosinusitis}

This subtype of chronic rhinosinusitis involves the inflammation of the maxillary sinusitis that is attributed to a fungal pathogen and can be classified into acute fungal rhinosinusitis, fungus balls or fulminant invasive fungal rhinosinusitis.

Acute fungal rhinosinusitis (AFRS), as described by Bent and Kuhn, is characterised by five properties [22]:

1. Nasal polyps.

2. Type I hypersensitivity to fungi (skin test or serum $\operatorname{IgE}$ testing).

3. Sample positive for fungal staining. 
4. Eosinophilic mucin that does not invade into sinus tissue.

5. Characteristic CT radiopaque sinus findings.

The predicted pathogenesis of AFRS is believed to be an allergic, immediate hypersensitivity reaction to an inhaled fungal organism resulting in a chronic inflammatory response in a predisposed individual, usually with asthma. The most common isolated fungal species include Bipolaris, Curvularia, Aspergillus, and Drechslera species.

Fungal balls are non-invasive, dense collections of fungal debris within the maxillary sinus. Found in mostly immunocompromised and elderly individuals, they are formed commonly of aspergillus, in response to prolonged exposure to the fungi through inhalation of airborne spores or oral antral communication.

Acute invasive fungal sinusitis is a rapidly infiltrating growth of the fungi with a high risk of morbidity and mortality. Immunocompromised individuals that are predisposed to this include those with:

- Diabetes,

- Leukaemia,

- Hard and soft tissue malignancy with neutropenia,

- Steroid therapy,

- Severely impaired cell-mediated immunity (congenital or acquired).

Initial clinical presentation involves an acute onset of facial/head pain, fever, epistaxis and, in severe cases, of bony erosion with extrasinus infiltration and possible mental status change. Urgent surgical management and post-operative antifungal therapy is required.

\subsection{Clinical Diagnosis}

Diagnosis of sinusitis is primarily through clinical history and examination with the possible adjuncts of imaging studies and/or laboratory testing. The signs and symptoms of sinusitis can be categorised in to major and minor. Major features include infraorbital and maxillary facial pain, pressure sensation, congestion/obstruction sensation, purulent rhinorrhoea, hyposmia and fever [23]. Minor features are present in some of the individuals with suspected sinusitis and include headache, fatigue, dental pain, halitosis, cough and ear pain/fullness.

The acute form of rhinosinusitis is indicated usually when a patient has recently suffered with the symptoms of an upper respiratory tract infection that is viral in nature. After an initial 7-10 day phase of viral infection with gradual recovery, patients give history of worsening symptoms. Evaluation of nasal discharges show secretions that were once clear at the at the initial stages of the viral infection that become yellow, green or grey in nature at the latter aspect of the biphasic disease process. Unilateral disease process provides greater indication towards sinusitis. Palpation over the infraorbital sinus region would elicit tenderness due to the pressure build-up from stasis and inflammation of the sinus cavity.

Patients with suspected chronic rhinosinusitis may present with symptoms of acute rhinosinusitis but to a milder degree, with the absence of a predisposing upper respiratory tract infection including fever. The presenting complaint may often be of the lack of effectiveness in acute medical management techniques [24]. Regardless of whether the causative factor in the chronicity of their sinusitis is from the presence of nasal polyps or not, patients will often present with facial pain or pressure, anterior or posterior nasal discharge and slight fatigue. Those that suffer from chronic sinusitis without the presence of polyps may also experience anosmia or hyposmia. Thus, the ambiguity in presentation makes the diagnosis of chronic sinusitis with clinical history and examination alone challenging, but nonetheless provide a vital role in their diagnosis and long-term management.

Endonasal examination in the form of anterior rhinoscopy may also be performed with headlighting and a speculum; alternatively with the large speculum of an otoscope. It can play a supportive role in visualising inflamed nasal mucosa, presence and quality of nasal discharge and the turbinates with an indication of nasal polyps or anatomical variation that may not have been suspected previously.

Nasal endoscopy provides an enhanced technique for direct visualisation of the nasal passage. Abnormalities in the nasal passage can be seen including the middle, superior turbinates and the osteomeatal mucociliary drainage passage. Where acute rhinosinusitis is diagnosed mainly through clinical history and simple examination, this method is more relevant for incidences of chronic rhinosinusitis. Nasal endoscopy can be used at 3, 6, 9 and 12 monthly intervals in chronic disease process to assess the degree of inflammation, discharge and size of nasal polyps.

Intraoral assessment should include assessment of both soft and hard tissues. The dentition requires close evaluation to rule out primary aetiology which can account for 5-10\% of acute rhinosinusitis cases [25]. Detailed assessment for extensive dental caries, periapical and/or periodontal infections for teeth distal to the canine is required. Where multiple teeth are unilaterally tender to percussion with a lack of correlation to dental pathology clinically or radiographically, sinusitis not of dental cause is a probable diagnosis. This sensation may also be elicited with facial or head movements. This is due to the close neural relationship between the maxillary sinus and upper molars. Pain or pressure sensation is also elicited from palpation in the most superior aspect of the maxillary buccal sulcus. In some cases, posterior discharge in to the nasopharynx that is either characteristically 
Table 23.3 Differential diagnosis for maxillary sinusitis, NICE guidelines 2018 [26]

- Upper respiratory tract infection.

- Allergic rhinitis.

- Adenoiditis or tonsillitis.

- Sinonasal tumour.

- Turbinate hypertrophy.

- Migraine.

- Giant cell arteritis.

- Temporomandibular joint dysfunction.

- Neuropathic or atypical facial pain.

clear or coloured in nature can be visualised during intraoral assessment.

Recurring episodes of acute rhinosinusitis (more than three episodes a year) requires wider consideration of the primary causative factor. Peters et al. has attributed recurrent acute rhinosinusitis to various reasons such as immunodeficiency, cystic fibrosis, ciliary dysfunction and anatomic abnormalities [16] (Table 23.3).

Severe episodes of acute rhinosinusitis originating from the maxillary sinuses are a rare presentation. Complications can arise with the involvement of the ethmoidal and frontal sinuses that are in close proximity to vital structures including the anterior cranial cavity and the orbits with associated venous drainage systems [27].

As per Ah- See et al. [28], certain features of sinusitis that require urgent intervention are:

- Bleeding.

- Proptosis/diplopia.

- Maxillary paresthesia.

- Intraorbital/intracranial complications.

- Osteomyelitis.

- Immunocompromised patients.

\subsection{Clinical Imaging}

Clinical imaging is a useful adjunct to the diagnosis of sinusitis. It can provide confirmatory and characteristic information to guide management of the condition.

Plain radiography is an accessible and cost-effective means of visualising the maxillary sinus. An orthopantomogram (OPG), though focusing on the dentition, maxilla and mandible, also includes a significant proportion of the maxillary sinuses and provides an opportunity to identify the presence of pathological features. Indication of sinusitis would be from the presence of unilateral or bilateral generalised diffuse radiopacity within the maxillary sinus. Causative disease process can also be visualised including the proximity of periapical pathology from the maxillary dentition, periodontal disease, cystic lesions of dental origin, foreign body presence, sinonasal mucocele or polyps. The significant level of artefact from overlying structures however limits the usefulness of this imaging modality. Where dental disease is a suspected contributing factor, dental periapical radiographs can be considered for a targeted and enhanced dental evaluation. Consequently, a dental cause for sinusitis can be challenging to diagnose, in particular in cases of chronic sinusitis.

The occipitomental view, also known as the 'Water's view, can additionally offer visualisation of the entire sinus as well as other paranasal sinuses, with fluid collection and mucosal thickening evident at varying angles. Care must be taken to differentiate between a benign mucosal cyst and a fluid level. A diagram showing the difference in the appearances between the two is shown in Fig. 23.7.

Despite the efficiency with which these images can be taken, one should consider whether the overall information gained for sinusitis diagnosis justifies the exposure to radiation. Plain imaging does not offer a comprehensive view of the maxillary sinus ostium, the occlusion of which will often be the causation of subsequent sinusitis. Apart from suggestion in the presence or absence of disease process, it does not offer information on the severity of the condition. With significant overlap, diagnosis of sinus masses can also be difficult.

Computer tomography (CT) (Fig. 23.8) offers the threedimensional visualisation of the sinuses and can be confirmatory in the presence or absence of sinus disease. These should be considered only where a patient's symptoms are vague or first-line medical management has thus far failed to offer significant relief of symptoms. Contrast medium is not required as they do not offer additional value towards diagnosis or management. It should also be noted that where CT scans of sinuses are taken, maxillary teeth should also be included to assess for their involvement in the disease process.

With appropriate manipulation of imaging sections, the location and severity of sinus disease can be gauged. Mucosal thickening is distinctly evident on CT scans, being more pronounced in cases of sinusitis with polyps. Further diagnostic information is available on the sinus content including airfluid levels. The patency of the sinus discharge tract can be assessed with detailed view of the osteomeatal complex on coronal sections thus allowing for surgical planning. The presence or absence of nasal masses can be confirmed in CT cases but can be used to differentiate between sinus polyps, cysts or tumours. Generalised thickening or sclerosis of the maxillary sinus walls may be indicative of chronic sinusitis whereby early infective processes cause demineralisation of the sinus wall and prolonged reactive host responses that result in sclerotic bone deposition. Where sclerosis is more localised in the maxillary sinus wall, there may be indication towards enquiry of previous sinus surgery.

Cone beam computer tomography (CBCT) can provide enhanced information on the pathological processes contrib- 
Fig. 23.7 Diagram showing the difference between the cyst/polyp fluid level and the mucosal
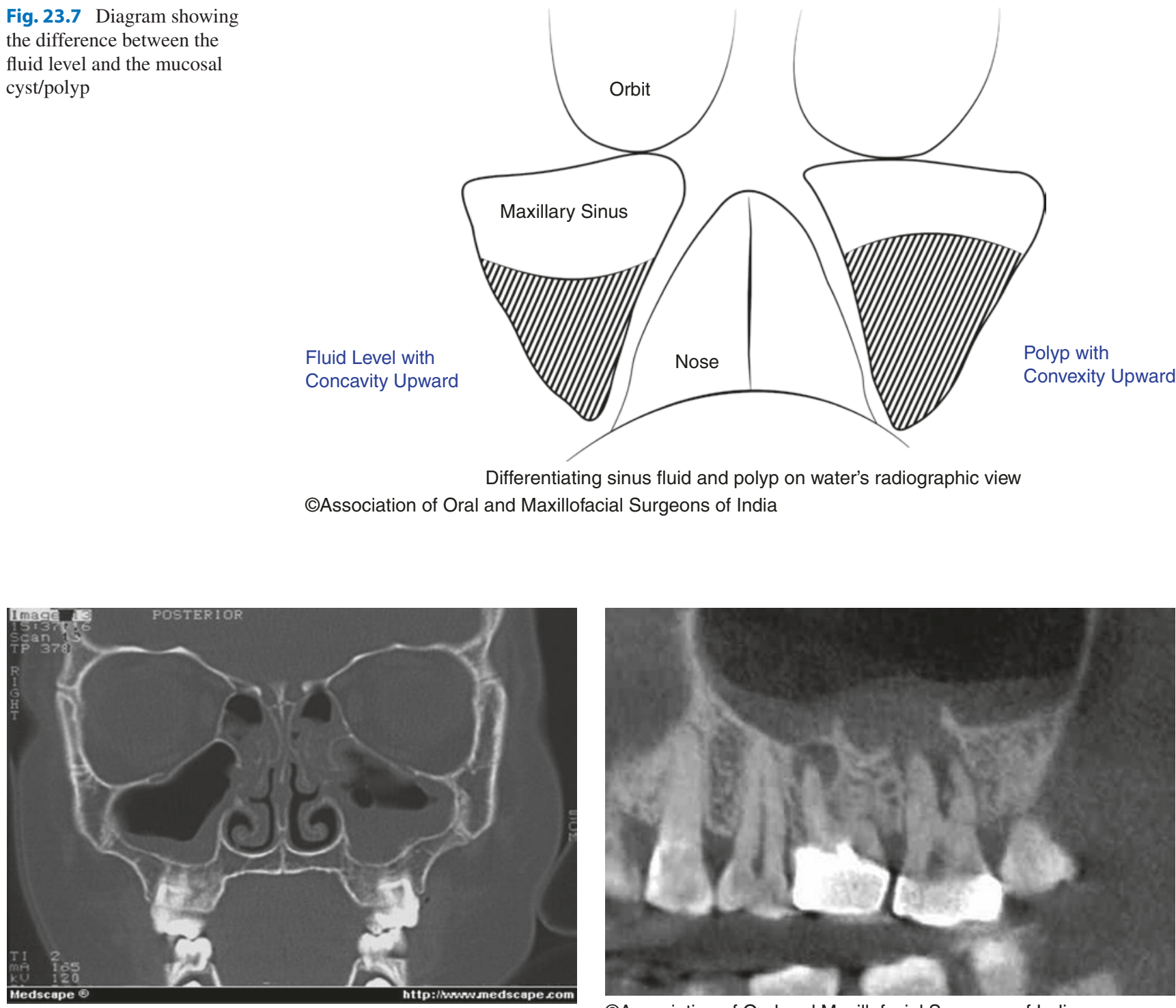

CAssociation of Oral and Maxillofacial Surgeons of India

Fig. 23.8 A coronal view of a CT scan showing thickened mucosal lining of the both maxillary sinus with a fluid level on the left maxillary sinus

uting towards sinusitis, with slices as fine as $0.4 \mathrm{~mm}$ as well as offering a lower radiation dose exposure, in the region of $10 \%$, in comparison to a fine slice CT scan (Fig. 23.9).

This is more relevant for cases of maxillary sinusitis of dental origin in which pathological processes can be tracked from the tooth to the sinus structure.

MRI scans offer an imaging modality that allows some enhanced interpretation in the appearance of the soft tissue within the sinus. This is not relevant in the majority of uncomplicated maxillary sinusitis presentations. The use of MRI is limited to cases where there is opacification of the maxillary sinus with no obvious obstruction, osseous abnormality or odontogenic pathology. It allows the differentiation between mucosal inflammation, complete fluid collection or a tumour within the maxillary sinus as well as its origin.

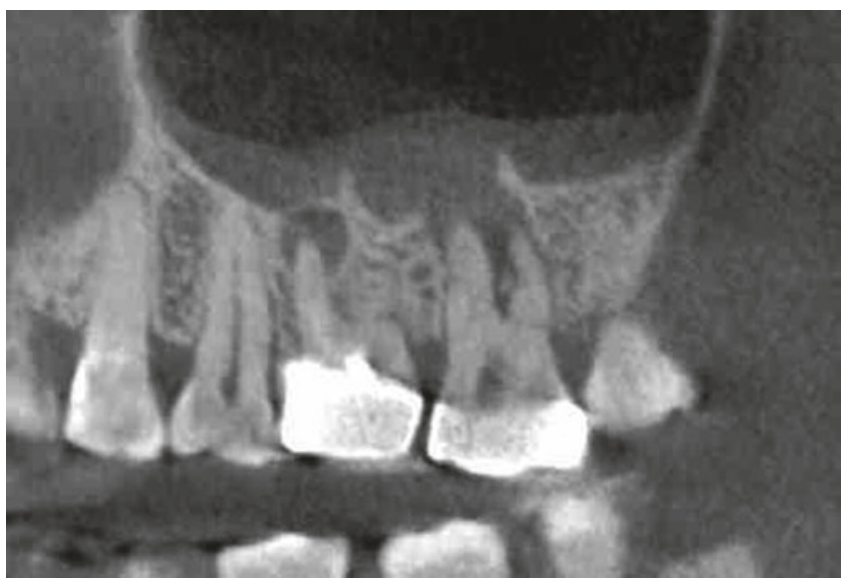

CAssociation of Oral and Maxillofacial Surgeons of India

Fig. 23.9 $\mathrm{CBCT}$ of the sinuses showing upper second molar with a breach in the periodontal ligament space and periapical area opening into the maxillary sinus causing sinusitis-a classic odontogenic sinusitis

\subsection{Treatment}

\subsubsection{Conservative Management}

In acute sinusitis cases, with a primary virus and secondary bacterial infection, the aim of treatment focuses around relieving of symptoms including pain, nasal congestion and discharge during the 2-3 week period that it will take to resolve. Antibiotics in the early stages are not recommended. Advice can be offered in the management of systemic virus infection including physical rest, adequate analgesia and increased fluid intake.

The pain sensation from the maxillary sinus region is attributed to pressure changes caused by occlusion of the 
ostia and thus, treatment to promote unblocking will inherently contribute towards pain reduction. During this process, analgesia in the form of paracetamol and ibuprofen can be utilised for management of pain.

Nasal saline and decongestants have been shown in some literature to be an effective means of opening the ostia and ecouraging ventilation.

Nasal saline mists and irrigations can offer a breakdown of nasal blockages, dilution of irritants and allergens, reduction of postnasal drainage and overall more effective mucociliary clearance [29]. Lavage/irrigation of the nasal passage can be performed using small quantities of salt or bicarbonate dissolved in warm water. These can be administered by inhaling small volumes of the self-made formulation in cupped hands through each nostril until nasal ventilation feels clearer. Alternative over-the-counter devices such as squeeze-bottles and syringes can be used. Humidified air and steam inhalation can also be utilised as a means of nasal secretory blockage breakdown. These irrigative techniques can offer supportive relief to other symptomatic management methods but are not deemed effective as monotherapy [30].

Where there is indication of chronic sinusitis, potential risk factors should be identified and addressed, including avoidance of triggers that may exacerbate allergies and asthmatic flare-ups. Where applicable, patients should be encouraged to stop smoking or being exposed to passive smoke.

\subsubsection{Medical Management}

Where acute sinusitis symptoms last for longer than 10 days, medical treatment modalities can be offered.

\subsubsection{Nasal Decongestants}

Topical nasal decongestants can be offered as a means of improving sinonasal ventilation through reducing mucosal secretion, nasal congestion and thus improved patency of the osteomeatal complex. Topical formulations can include phenylephrine $(0.25 \%)$, oxymetazoline $(0.5 \%)$ and xylometazoline [31]. These should be limited to use twice a day for up to 3 days. These formulations have a sympathomimetic effect causing vasoconstriction with subsequent reduced fluid secretion and mucosal inflammation. Longterm use of these agents can produce a rebound effect with the absence of sustained vasoconstriction.

\subsubsection{Topical Nasal Glucocorticosteroids}

Topical intranasal glucocorticosteroids may be considered in the management of prolonged acute rhinosinusitis or chronic rhinosinusitis as an adjunctive or monotherapy prior to the consideration of antibiotic prescription [32]. These can be utilised for up to 3 months in adult patients depending on the formulation used and especially where allergy is deemed a significant risk factor. Common formulations utilised include budesonide, ciclesonide, fluticasone furoate, fluticasone propionate, mometasone furoate and triamcinolone acetonide. These have been deemed an effective means of clearing nasal and sinus air passages in several studies when used in a retroclined, head tilt action to encourage exposure towards the middle meatus.

\subsubsection{Systemic Glucocorticosteroids}

The use of systemic glucocorticosteroids has been reserved for refractory cases of chronic rhinosinusitis, in particular those with allergy [2], as well as initial treatment for patients with suspected allergic fungal rhinosinusitis. It can provide symptomatic relief through improving ventilation through the sinuses, reducing the size of polyps and thus restoring some sense of smell. Current British guidelines suggest prednisolone $0.5 \mathrm{mgk} / \mathrm{kg}$ for 5-10 days as well as the adjunct therapy with betamethasone nasal drops [33], taking relevant consideration of those already on steroids for other conditions.

\subsubsection{Antimicrobial Therapy}

There is limited evidence to support the use of antibiotics in the short term for acute rhinosinusitis. A Cochrane review of 10 trials based in the primary care environment found that irrespective of treatment modality with or without antibiotics, $71 \%$ of patients' symptoms of uncomplicated acute sinusitis had resolved by the 2-week mark [34]. Where symptoms of sinusitis arise, the microbes of dominance usually include aerobic streptococcus pneumonia, haemophilus influenza and Moraxella catarrhalis. Antibiotic therapy should be reserved for cases of acute exacerbations of rhinosinusitis where symptoms haven't resolved after 7-10 days or for severe cases involving signs of systemic spread including fever and severe unilateral facial pain. Though the evidence is limited, some literature supports the use of amoxicillin for 7-14 days. This is mostly effective; however, approximately $20-30 \%$ of haemophilus influenza strains have been found to be resistant to amoxicillin due to the production of lactamase [35]. During persistent periods of acute sinusitis or during exacerbations of chronic sinusitis, there is a shift towards a mixture of anaerobic and aerobic pathogenesis. The Infectious Diseases Society of America recommends amoxicillin clavulanate as the first choice of antibiotic for 5-7 days [36], with clindamycin, doxycycline, levofloxacin or moxifloxacin for those with an allergy to penicillin. This can be used in combination with topical glucocorticosteroids to attain more efficient symptom relief [32]. In cases of chronic sinusitis, use of low-dose macrolides has shown some effectiveness [14]. In all cases, where viable, the choice of antibiotic should be guided by microbiological culture growth, attained from discharges from the middle meatus or surgically guided sample collection. 


\subsubsection{Surgery}

Surgical intervention is routinely carried out by Ear, Nose and Throat speciality and is usually indicated in the failed medical management of chronic sinusitis or as the first treatment for acute fungal rhinosinusitis. The aim of surgery is to attain normal function through restoration of ventilation and allowing physiological mucociliary drainage of the sinuses. This can be through opening and widening of the osteomeatal complex, removing foreign body and polypoid tissue, clearing the sinus of infected mucin, removal of chronically inflamed mucosal and bony tissue whilst throughout preserving as much of the virgin, healthy mucous membrane in the sinus as possible.

Extra-oral or intra-oral approaches can be taken. The classically used internal access is the Caldwell-Luc approach. George W Caldwell first published this technique in the New York Medical Journal in 1893, where he utilised the canine fossa approach to gain access to the sinus and perform intranasal drainage, significantly improving surgical outcomes. Henry Luc, a french surgeon, further adapted this method in 1897 for surgical treatment of chronic sinusitis where performed antrstomy in the middle meatus whilst Caldwell had performed an inferior meatal antrostomy.

\subsubsection{Surgical Method: Caldwell-Luc Approach}

Advanced imaging like CT or CBCT should be used for the procedure. Particular awareness is required for the presences of any septae that may interfere with the Caldwell-Luc access in to the sinus. In addition, assessment is required of the availability of a window on the anterior wall of maxillary sinus, with short maxillary sinus height creating challenges in this approach.

This procedure can be done either under a general or a local anaesthetic with sedation support; though complete painrelief and anaesthesia with the latter option is difficult to achieve (Fig. 23.10). Once adequate anaesthesia has been achieved, the upper lip is retracted an incision is made 2-3 $\mathrm{mm}$ above the mucogingival junction, parallel to the occlusal surface of the teeth. A full-thickness mucoperiosteal flap can be raised with a molt"s periosteal elevator to reveal the underlying bone. Often, bulbosities caused by the root projections of the upper canines and premolars are noticeable and they can be used as anatomical landmarks to avoid damaging the roots of the teeth. The periosteum can be raised elevated all the way to infraorbital foramen and care must be taken not to stretch the infraorbital nerve. Then a bony window can be made with a Rosehead bur 2-3 $\mathrm{mm}$ above the root apices of the teeth. On entry in to the sinus, a microbiology swabbing of the sinus content is done for culture and sensitivity. Once the swab has been taken, this is followed by stripping of the sinus lining, espe-

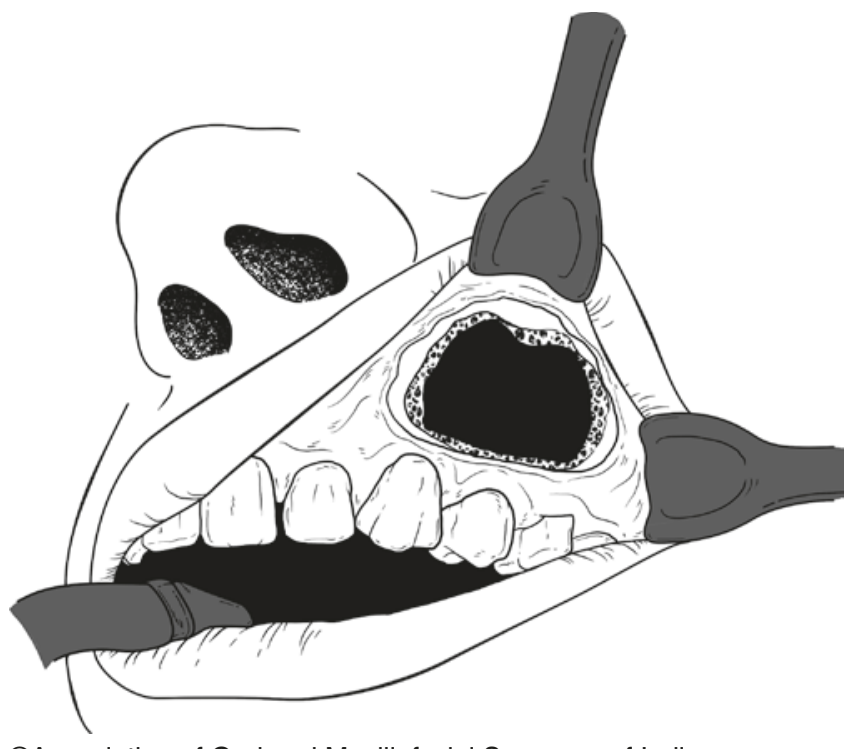

CAssociation of Oral and Maxillofacial Surgeons of India

Fig. 23.10 A diagram showing the intraoral approach for the CaldwellLuc technique

cially when treating chronic, persistant sinusitis. The lining can be removed with a microdebrider, Freer's elevator and curettes. Care must be taken not to remove tissue from the roof of the sinus due to the fact infraorbital nerve can be seen through the canal resorbed by maxillary sinusitis.

Any soft tissue curetted from the sinus can be sent for histopathological examination. A 30 or 45 degree endoscope is used to visualise the floor of the sinus which is not feasible with a standard 0 degree FESS. To complete the procedure, an intranasal antrostomy can be done to improve the drainage of the sinus. This procedure can be done by passing a curved haemostat through the nasal aperture to penetrate the lateral nasal wall, $1-1.5 \mathrm{~cm}$ posterior to the anterior attachment of the inferior nasal turbinate. Care should be taken to avoid injuring the opening of nasolacrimal duct. The projection of the haemostat can be visualised with an endoscope in the sinus simultaneously. Forceps are used to remove the fragments of bone created from the enlargement of the ostium and thus complete the intranasal antrostomy. The intra-oral Caldwell-Luc access is used to provide a final saline-wash within the sinus, prior to achieving haemostasis and would closure, often with resorbable sutures.

\subsubsection{Complications with Caldwell-Luc approach}

Complications associated with this surgical technique include infraorbital nerve parasthesia that is often transient and may last up to 6 months. Similarly there may be numbess of the attached gingivae and associated teeth or even possible devitalisation. If the lacrimal apparatus has been encountered, there may also be the risk of dacryocystitis. 

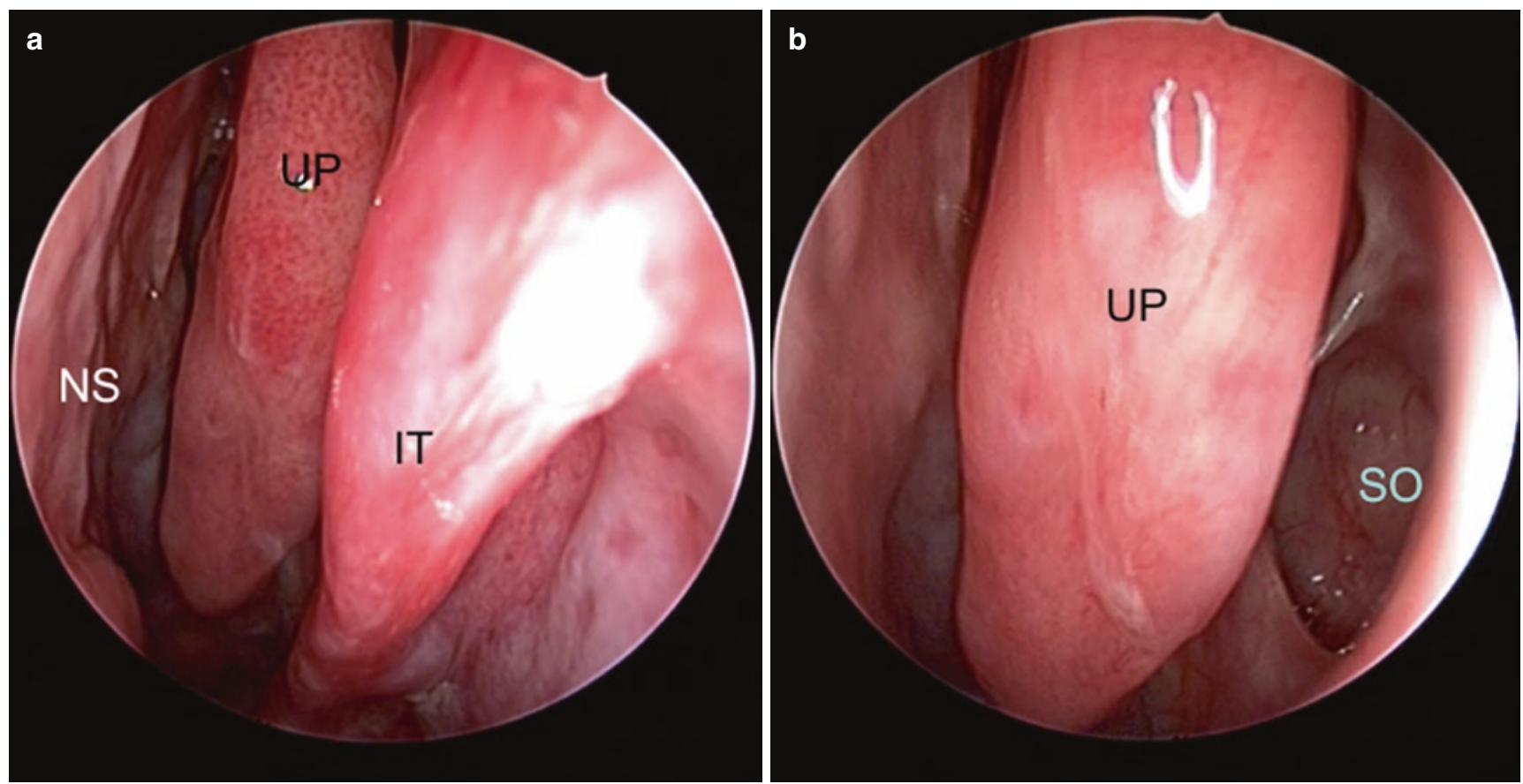

(c)Association of Oral and Maxillofacial Surgeons of India

Fig. 23.11 (a and b) Naso endoscopic pictures showing as the scope enters the left nostril. Nasal septum (NS) on the left and Inferior turbinate (IT) on the right and the uncinate process (UP). Further up showing maxillary sinus ostium (SO) and Uncinate process (UP)

\subsubsection{Surgical Method: Functional Endoscopic Sinus Surgery}

With technology continually advancing, many prospective studies report functional endoscopic sinus surgery (FESS) (Fig. 23.11) to be a safe and effective means of managing chronic sinusitis. For the FESS approach to be an effective surgical method in the management of chronic sinusitis, appropriate patient and surgical instrumentation related factors require consideration. Standard preparation for FESS involves an oral endotracheal tube which can be positioned based on surgeon's convenience to improve the access. Prior to donning surgical scrubs, the neuro-patties soaked in a vasoconstrictor solution may be placed along the floor and middle portion of the nasal cavity such that it is effective at the start of the operation. In addition, all Encoscopic acumen should be ready for the beginning of the procedure. Endoscopic instrumentation includes a 0, 30, and 70 degree angle scope, a powered debrider with a $4 \mathrm{~mm}$ straight and a 60 degree cannula, $4 \mathrm{~mm}$ long curved suction, various types of punch forceps, curettes and a monopolar suction cautery.

Before the start of the procedure, neuro-patties are removed and 1\% lidocaine and 1:100000 adrenaline injected into the septum, middle turbinate and uncinate process for the maxillary sinus-related issues. To survey the width and depth of the nasal cavity and also to establish the boundaries to avoid any complications, a systematic examination of the nasal cavity carried out with a 30 degree scope attached to an endoscopic tower/viewing screen. All the nasal structures should be visualised including posterior nasal choana and the inferior turbinates.

Systematic intranasal survey with the scope is also carried out as a part of the surgical planning to identify any nasal septal spur, septal deviations and turbinate hypertrophy to improve the access and to prevent any complications. Additionally, any polyps can be removed to improve the access. An angled probe is used to reflect the uncinate process to visualise hiatus semilunaris and infundibulum. Back fracturing the uncinate process finally allows visualisation of the maxillary sinus ostium and medial wall of the orbit. A $4 \mathrm{~mm}$ trimmer can be used to remove uncinate and part of the middle turbinate to gain access to maxillary sinus ostium. The superior border of the sinus ostium is an important anatomical landmark as it is the junction of the medial wall of the orbit and the lamina papyracea. At this point of dissection, a decision can be taken to do a minimal or extensive dissection process, and this depends on the extent of the disease process. Mostly, a minimal dissection (expansion of the ostium) is all that is necessary.

\subsubsection{Complications with the FESS Approach}

FESS is an essentially safe procedure in the hands of a trained and experienced surgeon; however there are a wide range of risks associated with this procedure due to the proximity of anatomical structures such as the orbits and their content, major arterial and venous structures and the base of the skull. Severe complications can include orbital content penetration that may lead to fat herniation, enopthalmous 
and damage to extraocular muscles; penetration in to the skull base can result in cerebrospinal fluid leakage and carotid artery dissection may cause a stroke or even death. Orbital content penetration leading to fat herniation, enophthalmos and damage to extraocular muscles. Crusting, septal perforation, mild bleeding, nasal obstruction, anosmia, minimal fat herniation from orbital wall perforation and crusting of the nasal mucosa are examples of what are believed to be minor complications.

Sinus surgery should be supported with medical management post-operatively to prevent further inflammation developing, particularly in the case of polyp removals that may re-establish without maintenance therapy.

\subsection{Conclusion}

Sinusitis can be a debilitating condition for patients whether acute or chronic. With close proximity to the dentition, symptoms of dental pain can result in presentation to the maxillofacial and dental professional. A succinct clinical history and examination is vital in attaining an accurate diagnosis. Though management is guided by diagnosis, management should commence with symptom relief.

Acknowledgements We would like to acknowledge Mr. Martyn Barnes and SurgTec for the endoscopic pictures attached to this chapter. More endoscopic images and videos can be accessed from www.surgtech.net website.

\section{References}

1. Lawson W, Patel ZM, Lin FY. The development and pathologic processes that influence maxillary sinus pneumatization. Anat Rec: Advances in Integrative Anatomy and Evolutionary Biology: Advances in Integrative Anatomy and Evolutionary Biology. 2008 Nov;291(11):1554-63.

2. Ugincius P, Kubilius R, Gervickas A, Vaitkus S. Chronic odontogenic maxillary sinusitis. Stomatologija. 2006;8:44-8.

3. Pommer B, Ulm C, Lorenzoni M, Palmer R, Watzek G, Zechner W. Prevalence, location and morphology of maxillary sinus septa: systematic review and meta-analysis. J Clin Periodontol. 2012 Aug;39(8):769-73.

4. Stallman JS, Lobo JN, Som PM. The incidence of concha bullosa and its relationship to nasal septal deviation and paranasal sinus disease. Am J Neuroradiol. 2004 Oct 1;25(9):1613-8.

5. Benninger MS. Rhinitis, sinusitis and their relationship to allergy. Am J Rhinol. 1992;6:37-43.

6. Aust R, Stierna P, Drettner B. Basic experimental studies of ostial patency and local metabolic environment of the maxillary sinus. Acta Otolaryngol Suppl. 1994;515:7-10. Discussion 1

7. ENT UK and Royal College of Surgeons. Commissioning guide: chronic rhinosinusitis. London: ENT UK and Royal College of Surgeons; 2016.

8. Pawankar R. Nasal polyposis: an update. Curr Opin Allergy Clin Immunol. 2003 Feb 1;3(1):1-6.
9. Norlander T, Fukami M, Westrin KM, Stierna P, Carlsöö B. Formation of mucosal polyps in the nasal and maxillary sinus cavities by infection. Otolaryngol Head Neck Surg. 1993 Sep;109(3):522-9.

10. Drake-Lee AB, McLaughlan P. Clinical symptoms, free histamine and $\mathrm{IgE}$ in patients with nasal polyposis. Int Arch Allergy Appl Immunol. 1982;69:268-71.

11. Gwaltney JM Jr, Scheld WM, Sande MA, Sydnor A. The microbial etiology and antimicrobial therapy of adults with acute communityacquired sinusitis: a fifteen-year experience at the University of Virginia and review of other selected studies. J Allergy Clin Immunol. 1992;90:457-61. Discussion 62

12. Finegold SM, Flynn MJ, Rose FV, et al. Bacteriologic findings associated with chronic bacterial maxillary sinusitis in adults. Clin Infect Dis. 2002;35:428-33.

13. Brook I, Foote PA, Hausfeld JN. Increase in the frequency of recovery of methicillin-resistant Staphylococcus aureus in acute and chronic maxillary sinusitis. J Med Microbiol. 2008;57:1015-7.

14. Fokkens WJ, Lund VJ, Mullol J, et al. European position paper on rhinosinusitis and nasal polyps 2012. Rhinol Suppl. 2012;(23):3 p preceding table of contents, 1-298.

15. Serrano E, Neukirch F, Pribil C, Jankowski R, Klossek JM, Chanal I, et al. Nasal polyposis in France: impact on sleep and quality of life. J Laryngol Otol. 2005;119(7):543-9.

16. Peters AT, Spector S, Hsu J, Hamilos DL, Baroody FM, Chandra RK, Grammer LC, Kennedy DW, Cohen NA, Kaliner MA, Wald ER. Diagnosis and management of rhinosinusitis: a practice parameter update. Ann Allergy Asthma Immunol. 2014 Oct $1 ; 113(4): 347-85$.

17. Koskinen OM, Husman TM, Meklin TM, Nevalainen AI. The relationship between moisture or mould observations in houses and the state of health of their occupants. Eur Respir J: Official Journal of the European Society for Clinical Respiratory Physiology. 1999 Dec;14(6):1363-7.

18. Report of the rhinosinusitis task force committee meeting Alexandria, Virginia, August 17, 1996. Otolaryngol Head Neck Surg. 1997;117(3 Pt 2):S1-68.

19. Vestin Fredriksson M, Öhman A, Flygare L, Tano K. When maxillary sinusitis does not heal: findings on CBCT scans of the sinuses with a particular focus on the occurrence of odontogenic causes of maxillary sinusitis. Laryngoscope Investig Otolaryngol. 2017 Dec;2(6):442-6.

20. Patel NA, Ferguson BJ. Odontogenic sinusitis: an ancient but underappreciated cause of maxillary sinusitis. Curr Opin Otolaryngol Head Neck Surg. 2012 Feb 1;20(1):24-8.

21. Arias-Irimia O, Barona-Dorado C, Santos-Marino JA, MartínezRodríguez N, Martínez-González JM. Meta-analysis of the etiology of odontogenic maxillary sinusitis. Med Oral Patol Oral Cir Bucal. 2010 Jan 1;15(1):e70-3.

22. Bent JP III, Kuhn FA. Allergic fungal sinusitis/polyposis. Allergy Asthma Proc. 1996;17:259-68.

23. Rosenfeld RM, Andes D, Bhattacharyya N, et al. Clinical practice guideline: adult sinusitis. Otolaryngol Head Neck Surg. 2007;137(suppl):S1-S31.

24. Tan BK, Chandra RK, Pollak J, et al. Incidence and associated premorbid diagnoses of patients with chronic rhinosinusitis. J Allergy Clin Immunol. 2013;131:1350-60.

25. Williams JW, Simel DL. Does this patient have sinusitis? Diagnosing acute sinusitis by history and physical examination. JAMA. 1993;270:1242-6.

26. National Institute for Health and Care Excellence. Sinusitis. London: NICE; 2018.

27. Cummings CW, Haughey BH, Thomas JR, Harker LA, Flint PW, Haughey BB, et al. Cummings otolaryngology: head \& neck surgery. 4th ed. Philadelphia: Mosby; 2005. 
28. Ah-See KW, Evans AS. Sinusitis and its management. BMJ: Bri Med J. 2007 Feb 17;334(7589):358.

29. Hauptman G, Ryan MW. The effect of saline solutions on nasal patency and mu- cociliary clearance in rhinosinusitis patients. Otolaryngol Head Neck Surg. 2007;137:815-21.

30. Harvey R, Hannan SA, Badia L, Scadding G. Nasal saline irrigations for the symptoms of chronic rhinosinusitis. Cochrane Database Syst Rev. 2007;3:CD006394.

31. Kretzschmar DP, Kretzschmar CJ. Rhinosinusitis: review from a dental perspective. Oral Surg Oral Med Oral Pathol Oral Radiol Endod. 2003 Aug 1;96(2):128-35.

32. Zalmonovici A, Yaphe J. Steroids for acute sinusitis. Cochrane Database Syst Rev. 2007;(2):CD005149.
33. Scadding GK, Durham SR, Mirakian R, Jones NS, Drake-Lee AB, Ryan D, et al. BSACI guidelines for the management of rhinosinusitis and nasal polyposis. Clin Exp Allergy. 2008;38:260-75.

34. Lemiengre MB, van Driel ML, Merenstein D, Young J, De Sutter AI. Antibiotics for clinically diagnosed acute rhinosinusitis in adults. Cochrane Database Syst Rev. 2012;10:CD006089.

35. Fairbanks DN. Inflammatory diseases of the sinuses: bacteriology and antibiotics. Otolaryngol Clin N Am. 1993;26:549-59.

36. Chow AW, Benninger MS, Brook I, et al. IDSA clinical practice guideline for acute bacterial rhinosinusitis in children and adults. Clin Infect Dis. 2012;54:e72-e112.

Open Access This chapter is licensed under the terms of the Creative Commons Attribution 4.0 International License (http://creativecommons. org/licenses/by/4.0/), which permits use, sharing, adaptation, distribution and reproduction in any medium or format, as long as you give appropriate credit to the original author(s) and the source, provide a link to the Creative Commons license and indicate if changes were made.

The images or other third party material in this chapter are included in the chapter's Creative Commons license, unless indicated otherwise in a credit line to the material. If material is not included in the chapter's Creative Commons license and your intended use is not permitted by statutory regulation or exceeds the permitted use, you will need to obtain permission directly from the copyright holder. 